\title{
BMJ Open Prevalence and risk factors associated with prehypertension in Shunde District, southern China
}

Yuli Huang, ${ }^{1,2}$ Wenke Qiu, ${ }^{3}$ Changhua Liu, ${ }^{1}$ Dingji Zhu, ${ }^{1}$ Jinghai Hua, ${ }^{1}$ Xiaoyan Cai, ${ }^{2}$ Yanxian $\mathrm{Wu},{ }^{2}$ Yunzhao $\mathrm{Hu}^{2}{ }^{2}$ Dingli $\mathrm{Xu}^{1}$

To cite: Huang Y, Qiu W, Liu C, et al. Prevalence and risk factors associated with prehypertension in Shunde District, southern China. BMJ Open 2014;4:e006551. doi:10.1136/bmjopen-2014006551

- Prepublication history for this paper is available online. To view these files please visit the journal online (http://dx.doi.org/10.1136/ bmjopen-2014-006551).

$\mathrm{YH}$ and $\mathrm{WQ}$ contributed equally to this work.

Received 4 September 2014 Revised 8 October 2014 Accepted 27 October 2014

CrossMark

${ }^{1}$ Department of Cardiology, Nanfang Hospital, Southern Medical University, Guangzhou, People's Republic of China

${ }^{2}$ Department of Cardiology, The First People's Hospital of Shunde, Foshan, People's Republic of China

${ }^{3}$ Department of Health Management Center, The First People's Hospital of Shunde, Foshan, People's Republic of China

\section{Correspondence to}

Professor Dingli Xu; dinglixu@fimmu.com and Professor Yunzhao Hu; huyunzha04406@163.com

\section{ABSTRACT}

Objective: To explore the prevalence and combined cardiovascular risk factors of prehypertension in southern China.

Design: A retrospective study; the logistic regression model was used to find the risk factors of prehypertension.

Setting: The study was conducted in Shunde District, southern China, using the community-based health check-up information.

Participants: Participants aged $\geq 35$ years with complete health check-up information data between January 2011 and December 2013 were enrolled and divided into hypertension, prehypertension and optimal blood pressure (BP) groups. Prehypertension was further divided into low-range (BP 120-129/80-84 mm Hg) and high-range (BP 130-139/85-89 mm Hg) subgroups.

Outcome measures: The prevalence of prehypertension and the combined cardiovascular risk factors within the prehypertensive subgroups.

Results: Of the 5362 initially reviewed cases (aged $\geq 35$ years), 651 were excluded because of missing data. The proportions of optimal BP, prehypertension and hypertension were $39.1 \%, 38.6 \%$ and $22.3 \%$, respectively. The average age, proportion of male sex, overweight, impaired fasting glucose (IFG), dyslipidaemia and hyperuricaemia were significantly higher in the prehypertension group than in the optimal BP group (all $p<0.05$ ). Compared with low-range prehypertension, the proportions of overweight, dyslipidaemia and IFG were higher in the high-range prehypertension group (all $p<0.05)$. Multivariate logistic regression analysis showed that overweight ( $\mathrm{OR}=2.84,95 \% \mathrm{Cl} 1.55$ to 5.20 ), male sex ( $O R=2.19,95 \% \mathrm{Cl} 1.39$ to 3.45 ), age (per 10 years, $\mathrm{OR}=1.21,95 \% \mathrm{Cl} 1.02$ to $1.44, \mathrm{p}=0.03)$ and

hyperuricaemia ( $\mathrm{OR}=1.70,95 \% \mathrm{Cl} 1.14$ to 2.54 ) were independent risk factors of prehypertension.

Conclusions: Prehypertension is highly prevalent in southern China. Prehypertensive individuals presented with many other cardiovascular risk factors. There was heterogeneity of combined risk factors within the prehypertensive subgroups.

\section{INTRODUCTION}

In 2003, the seventh report of the Joint National Committee on Prevention, Detection,

\section{Strengths and limitations of this study}

- This study shows that prehypertension is highly prevalent in the Shunde District, southern China.

- With the economic development and lifestyle changes in China, obesity/overweight has become a very important risk factor for increased blood pressure.

- This is the first study to show that there was a significant heterogeneity of combined risk factors within the prehypertensive subgroups.

- Some important confounding factors possibly associated with increased blood pressure were not evaluated in the present study.

Evaluation, and Treatment of High Blood Pressure (JNC 7) proposed a new blood pressure (BP) category, including 120-139 $\mathrm{mm} \mathrm{Hg}$ systolic BP (SBP) or $80-89 \mathrm{~mm} \mathrm{Hg}$ diastolic BP (DBP), designated as 'prehypertension'. ${ }^{1}$ The prevalence of prehypertension is up to $30-50 \%$ worldwide, as well as in many districts of China. ${ }^{2-4}$ It is known that in China the prevalence of hypertension is significantly higher in the northern area than in the southern area because of the colder climate and high sodium intake. ${ }^{5}$ Such regional factors may also affect the prevalence of prehypertension. However, the prevalence of prehypertension in Guangdong Province, southern China has been rarely reported.

Prehypertensive individuals are prone to progress into frank hypertension, and most of them present with clustering of other cardiovascular risk factors. ${ }^{6-8}$ However, the use of the term 'prehypertension' is still controversial. Most of the arguments against using this term consist of the possible public anxiety and overtreatment it may cause. Further, there is a high heterogeneity within this category because the risk of progressing to hypertension and developing cardiovascular disease (CVD) is higher among people with BP 130-139/85-89 $\mathrm{mm} \mathrm{Hg}$ than among those with BP 120-129/80-84 mm Hg. ${ }^{6}{ }^{9}$ Furthermore, the 
question of whether the concurrent cardiovascular risk factors in subgroups of prehypertension are different remains unanswered.

Given these inconsistent results, we conducted a retrospective analysis to explore the prevalence of prehypertension, and the cardiovascular risk factors in the subgroups of prehypertension in Guangdong Province, southern China.

\section{METHODS}

\section{Study participants}

We performed a retrospective study in Shunde District, a traditional but economically developed district of Guangdong Province, in China, using the communitybased health check-up information. Community-based health check-up information was collected in the Health Management Center of the First People's Hospital of Shunde. The centres provided data for participants who enrolled in their health check-up programmes conducted between January 2011 and December 2013. Participants aged $\geq 35$ years with complete data for the following characteristics were included in this study: age, sex, smoking/drinking habits, history of chronic diseases and treatment, family history of hypertension, height, weight, $\mathrm{BP}$, fasting plasma glucose (FPG), total cholesterol (TC), triglycerides (TG), low-density lipoprotein-cholesterol (LDL-C), high-density lipoprotein-cholesterol (HDL-C), serum creatinine $(\mathrm{Scr})$, blood urea nitrogen and serum uric acid (UA).

\section{BP measurement}

Although our data were based on retrospective analysis of community-based health check-up information, the protocol of BP measurement in our Health Management Center is carried out consistently since the foundation of the department. Participants were asked to avoid caffeinated beverages, smoking and exercise for at least $30 \mathrm{~min}$, and BP measurements were taken after the participants were allowed to rest quietly for at least 5 min. Three BP measurements (2 min between each) were obtained for each individual by trained nurses, who were part of the Health Management Center, with a mercury sphygmomanometer. The first and fifth Korotkoff sounds were recorded as SBP and DBP, respectively. During the measurements, the participants were seated with the arm supported at the level of the heart. The mean of three BP measurements was calculated and recorded.

\section{Definition of correlative risk factors}

The correlative risk factors estimated in our study included the following: (1) BP classification was based on the recommendations from the JNC 7. ${ }^{1}$ Optimal BP was defined as $\mathrm{SBP}<120 \mathrm{~mm} \mathrm{Hg}$ and DBP $<80 \mathrm{~mm} \mathrm{Hg}$. Hypertension was defined as SBP $\geq 140 \mathrm{~mm} \mathrm{Hg}$ and/or DBP $\geq 90 \mathrm{~mm} \mathrm{Hg}$, or previously diagnosed as hypertension and currently undergoing antihypertensive treatment. Prehypertension was defined if individuals were not undergoing antihypertensive treatment and had an SBP of 120-139 $\mathrm{mm} \mathrm{Hg}$ and/or DBP of $80-89 \mathrm{~mm} \mathrm{Hg}$. Prehypertension was further divided into low-range (SBP 120-129 and/or DBP 80 $84 \mathrm{~mm} \mathrm{Hg}$ ) and high-range (SBP 130-139 $\mathrm{mm} \mathrm{Hg}$ and/or DBP $85-89 \mathrm{~mm} \mathrm{Hg}$ ) subgroups. (2) Impaired glucose regulation was diagnosed based on FPG according to the American Diabetes Association criteria, ${ }^{10}$ including diabetes mellitus (DM; FPG $\geq 7.0 \mathrm{mmol} / \mathrm{L}$ ) and impaired fasting glucose (IFG, FPG 5.6-6.9 mmol/L). Dyslipidaemia was defined as with a history of receiving antidyslipidaemia agents or TC $\geq 5.18 \mathrm{mmol} / \mathrm{L}$, LDL-C $\geq 3.37 \mathrm{mmol} / \mathrm{L}$, HDL-C $<1.04 \mathrm{mmol} / \mathrm{L}$ and/or TG $\geq 1.7 \mathrm{mmol} / \mathrm{L}$, according to the 2007 Guidelines for Prevention and Treatment of Dyslipidemia in Adults in China. ${ }^{11}$ Hyperuricaemia was defined as UA $\geq 416 \mu \mathrm{mol} / \mathrm{L}$ in men and $357 \mu \mathrm{mol} / \mathrm{L}$ in women. (3) Overweight and obesity were defined as body mass index (BMI) $24-27.9 \mathrm{~kg} / \mathrm{m}^{2}$ and BMI $\geq 28 \mathrm{~kg} / \mathrm{m}^{2}$ according to Chinese criteria. ${ }^{12}$ (4) The estimated glomerular filtration rate (eGFR) of each participant was estimated using the modified Modification of Diet in Renal Disease equation adapted for the Chinese ${ }^{13}$ as: $\mathrm{eGFR}=186 \times \mathrm{Scr}^{-1.154} \times \mathrm{age}^{-0.203} \times 0.742 \quad($ female $) \times 1.233$ (Chinese).

\section{Statistical analysis}

Statistical analysis was performed using the Statistical Package for the Social Science software release V.16.0 (SPSS Inc, Chicago, Illinois, USA). Continuous variables are presented as mean (SD) or median (IQR) as appropriate. Categorical variables are expressed as percentages. After testing for normality using the Kolmogorov-Smirnov test, continuous variables were compared using a t test or the Mann-Whitney $\mathrm{U}$ test, and categorical variables were compared by $\chi^{2}$ test or Fisher's exact test as appropriate. Multiple logistic regression analysis was performed to evaluate predictive factors for prehypertension. Individuals with optimal BP were used as the reference group. Multicollinearity (strong correlations among independent variables) was examined by collinearity diagnostic statistics. Variance inflation factor values $>2.5$ or tolerance $<0.4$ may indicate concern for multicollinearity in logistic regression models. ${ }^{14}$ A value of $\mathrm{p}<0.05$ was considered statistically significant.

\section{RESULTS}

\section{Prevalence of prehypertension}

Of the 5362 cases (aged $\geq 35$ years) initially reviewed, 651 were excluded because of missing data. Finally, 4711 cases (2674 men, 2037 women) were analysed. The proportions of optimal BP, prehypertension and hypertension were $39.1 \%$ (1842 cases), 38.6\% (1819 cases) and 22.3\% (1050 cases), respectively. The prevalence of prehypertension was higher in men than in women $(43.5 \%$ vs $32.2 \%$, $\mathrm{p}<0.001)$. There was an increasing trend of prehypertension prevalence associated with age (table 1 ). 
Table 1 Prevalence of prehypertension and hypertension by sex and age group

\begin{tabular}{|c|c|c|c|c|}
\hline Age (years) & $\mathbf{n}$ & Optimal BP (\%) & Prehypertension (\%) & Hypertension (\%) \\
\hline \multicolumn{5}{|l|}{ Male } \\
\hline $35-49$ & 970 & 48.1 & 34.4 & 17.4 \\
\hline $50-64$ & 996 & 27.1 & 48.0 & 24.9 \\
\hline$\geq 65$ & 708 & 20.6 & 49.6 & 29.8 \\
\hline Total & 2037 & 33.0 & 43.5 & 23.5 \\
\hline \multicolumn{5}{|l|}{ Female } \\
\hline $35-49$ & 758 & 59.8 & 26.5 & 12.9 \\
\hline $50-64$ & 797 & 42.3 & 36.3 & 22.2 \\
\hline$\geq 65$ & 482 & 35.1 & 34.4 & 30.5 \\
\hline Total & 2674 & 47.1 & 32.2 & 20.7 \\
\hline \multicolumn{5}{|l|}{ All } \\
\hline $35-49$ & 1728 & 53.2 & 31.0 & 15.5 \\
\hline $50-64$ & 1793 & 33.9 & 42.8 & 23.7 \\
\hline$\geq 65$ & 1190 & 26.5 & 43.4 & 30.1 \\
\hline Total & 4711 & 39.1 & 38.6 & 22.3 \\
\hline
\end{tabular}

\section{Risk factors clustering in different blood pressure statuses}

The average age, proportion of male sex, family history of hypertension, overweight, IFG, dyslipidaemia, hyperuricaemia, levels of FPG, TC, TG, BMI and UA were significantly higher in the prehypertension and hypertension groups than in the optimal $\mathrm{BP}$ group (all $\mathrm{p}<0.05$ ). The proportions of DM, obesity and level of LDL-C were also higher in the hypertension group than in the optimal BP group (all $\mathrm{p}<0.05$ ); however, the differences were not significant in the prehypertension group compared with that in the optimal BP group (table 2).

\section{Cardiovascular risk factors in different subranges of prehypertension}

To explore the heterogeneity within the prehypertension category, patients with prehypertension were further classified into low-range and high-range subgroups. The proportions of male sex, overweight, IFG, BMI and levels of FPG were higher in the low-range prehypertension group than in the optimal BP group (all $\mathrm{p}<0.05$ ), but there were no significant differences for other cardiovascular risk factors (all $\mathrm{p}>0.05$ ). The proportions of male sex, overweight, obesity, dyslipidaemia, DM, IFG, hyperuricaemia and BMI, and levels of TC, LDL-C, TG, FPG and UA were higher in the high-range prehypertension group than in the optimal BP group (all $\mathrm{p}<0.05$ ). Compared with low-range prehypertension, the proportions of overweight, dyslipidaemia and IFG were higher in the high-range prehypertension (all $\mathrm{p}<0.05$; table 3 ).

\section{Risk factors associated with prehypertension}

The multivariable-adjusted risk factors associated with prehypertension are presented in table 4. High BMI (overweight/obesity) was the most important risk factor for prehypertension $(\mathrm{OR}=2.84,95 \%$ CI 1.55 to 5.20 , $\mathrm{p}<0.001$ ). Age ( per 10 years, OR $=1.21,95 \%$ CI 1.02 to 1.44, $\mathrm{p}=0.03)$, male sex $(\mathrm{OR}=2.19,95 \%$ CI 1.39 to 3.45 , $\mathrm{p}<0.001)$ and hyperuricaemia $(\mathrm{OR}=1.70,95 \%$ CI 1.14 to 2.54, $\mathrm{p}=0.009$ ) were also significantly associated with prehypertension. Furthermore, collinearity statistics were $>0.4$ for tolerance and $<2.5$ for the variance inflation factor, suggesting that multicollinearity was not a concern among the independent variables.

\section{DISCUSSION}

In this study, we found that prehypertension is highly prevalent in the Shunde District, Guangdong Province. Prehypertensive individuals presented with other risk factors associated with CVD, such as overweight, dyslipidaemia, impaired glucose and hyperuricaemia. Furthermore, combined cardiovascular risk factors were more significant in people with high-range prehypertension. To the best of our knowledge, this is the first study to show that there was a significant heterogeneity of combined risk factors within the prehypertensive subgroups.

Many epidemiological studies have demonstrated that prehypertension is an important public health problem. However, the prevalence of prehypertension in different countries and districts differs significantly, and may be influenced by different regional factors, such as climate and lifestyle, as well as ethnicity. At the beginning of this century (2000-2001), a cross-sectional survey found that the prevalence of prehypertension was $21.9 \%$ among Chinese participants aged between 35 and 74 years. ${ }^{3}$ However, in other subsequent studies, the prevalence of prehypertension was significantly higher than this ratio. In rural northeastern China, the prevalence of prehypertension was $35.1 \%$ in men and $32.5 \%$ in women, ${ }^{15}$ and up to $40 \%$ in the whole population from urban areas of northeastern China, ${ }^{16}$ which may be associated with the cold climate and high sodium diet. In this study, we found that the prevalence of prehypertension in the Shunde District of Guangdong Province, a traditional but economically developed district of southern China, was up to $38.6 \%$. It was very similar to that in the urban areas of northeastern China, ${ }^{16}$ and significantly higher than that reported at the beginning of this century for the 
Table 2 Cardiovascular risk factors in different blood pressure statuses

\begin{tabular}{|c|c|c|c|c|}
\hline & Optimal BP $(n=1842)$ & Prehypertension $(n=1819)$ & Hypertension $(n=1050)$ & p Value \\
\hline Age (years) & $48.8 \pm 10.5$ & $52.1 \pm 11.3^{*}$ & $54.3 \pm 10.4^{*}$ & 0.008 \\
\hline Male (n (\%)) & $829(45.0)$ & $1163(63.9)^{*}$ & $628(59.8)^{*} \dagger$ & $<0.001$ \\
\hline Smoking (n (\%)) & $301(16.3)$ & $287(15.8)$ & $186(17.7)$ & 0.77 \\
\hline Alcoholic (n (\%)) & $35(1.9)$ & $42(2.3)$ & $27(2.6)$ & 0.464 \\
\hline $\begin{array}{l}\text { Family history of hypertension } \\
(\mathrm{n}(\%))\end{array}$ & $274(14.9)$ & $292(16.1)^{\star}$ & $203(19.3)^{\star} \dagger$ & 0.007 \\
\hline Overweight $(\mathrm{n}(\%))$ & $236(12.8)$ & $395(21.7)^{\star}$ & $241(23.0)^{*}$ & $<0.001$ \\
\hline Obesity (n (\%)) & $128(6.9)$ & $156(8.6)$ & $98(9.3)^{\star}$ & 0.019 \\
\hline BMI $\left(\mathrm{kg} / \mathrm{m}^{2}\right)$ & $21.6 \pm 3.2$ & $24.9 \pm 3.5^{*}$ & $25.6 \pm 4.8^{*}$ & 0.001 \\
\hline Dyslipidaemia (n (\%)) & $420(22.8)$ & $488(26.8)^{*}$ & $312(29.7)^{\star} \dagger$ & $<0.001$ \\
\hline $\mathrm{TC}(\mathrm{mmol} / \mathrm{L})$ & $5.02 \pm 1.25$ & $5.39 \pm 1.67^{*}$ & $5.64 \pm 1.59^{\star} \dagger$ & 0.01 \\
\hline LDL-C (mmol/L) & $3.21 \pm 1.15$ & $3.45 \pm 1.18$ & $3.68 \pm 1.32^{*}$ & 0.03 \\
\hline HDL-C (mmol/L) & $1.18 \pm 0.36$ & $1.09 \pm 0.38$ & $1.02 \pm 0.41$ & 0.75 \\
\hline $\mathrm{TG}(\mathrm{mmol} / \mathrm{L})$ & $1.66 \pm 0.42$ & $2.07 \pm 0.58^{\star}$ & $2.09 \pm 0.63^{*}$ & 0.001 \\
\hline $\mathrm{DM}(\mathrm{n}(\%))$ & $138(7.5)$ & $168(9.2)$ & $109(10.4)^{*}$ & 0.002 \\
\hline IFG (n (\%)) & $261(14.2)$ & $384(21.1)^{\star}$ & $275(26.2)^{*} \dagger$ & $<0.001$ \\
\hline $\mathrm{FPG}(\mathrm{mmol} / \mathrm{L})$ & $5.14 \pm 1.96$ & $5.79 \pm 2.03^{\star}$ & $5.91 \pm 2.27^{*}$ & 0.003 \\
\hline $\operatorname{Scr}(\mathrm{mmol} / \mathrm{L})$ & $72.2 \pm 19.7$ & $75.0 \pm 25.1$ & $79.2 \pm 28.9$ & 0.14 \\
\hline BUN (mmol/L) & $5.34 \pm 1.71$ & $6.01 \pm 1.87$ & $6.25 \pm 1.99$ & 0.35 \\
\hline eGFR $\left(\mathrm{mL} / \mathrm{min} / 1.73 \mathrm{~m}^{2}\right)$ & $126.8 \pm 27.9$ & $125.2 \pm 29.4$ & $118.1 \pm 28.6$ & 0.76 \\
\hline Hyperuricaemia (n (\%)) & $233(12.6)$ & $302(16.6)^{\star}$ & $226(21.5)^{\star} \dagger$ & 0.001 \\
\hline UA (mmol/L) & $377.5 \pm 32.2$ & $402.9 \pm 34.6^{*}$ & $428.1 \pm 33.8^{*} \dagger$ & 0.007 \\
\hline
\end{tabular}

entire country. ${ }^{3}$ Further analysis showed that increased BMI was the most important risk factor for prehypertension in our study. Even among patients with low-range prehypertension, BMI was significantly increased compared with the optimal BP group. Therefore, our study suggests that although sodium intake is relatively low in the Guangdong Province in southern China, ${ }^{17}$ the prevalence of prehypertension is almost as high as that in the northern area. With the economic development and lifestyle changes, obesity/overweight has become a very important risk factor for increased BP.

Although the proportions of IFG and dyslipidaemia were higher in the prehypertension group than in the optimal BP group, in multivariable analysis the associations of IFG and dyslipidaemia with prehypertension were not significant after adjustment for BMI. Many studies have documented that overweight/obesity can cause significant insulin resistance, which may play an important role in impaired glucose metabolism, dyslipidaemia and increased BP. ${ }^{18}{ }^{19}$ Clinical studies have shown that weight control can significantly lower $\mathrm{BP}^{20}$ These results indicated that lifestyle modifications, such as weight loss, are effective in the long-term primary prevention of hypertension. With the economic development and lifestyle changes, lifestyle modifications should be emphasised as a cornerstone in modern China.

In addition to the traditional risk factors, previous studies have found that serum UA levels were significantly associated with prehypertension. ${ }^{21} 22$ The mechanisms may be associated with inhibition of the nitric oxide pathway and activation of the renin-angiotensin system. Further, UA can cause a proliferation of vascular smooth muscle cells and renal microvascular damage because of local inflammation and oxidative stress, finally leading to high BP. ${ }^{23}$ In our study, we found that the level of UA tended to increase in the low-range prehypertension group $(\mathrm{p}=0.07)$, and the difference was significant between high-range prehypertension and the optimal BP groups. These results indicate that the effect of UA on BP may be increased throughout the entire prehypertension range.

In a recent randomised controlled trial, prehypertensive obese adolescents aged 11-17 years were enrolled and randomised to a urate-lowering therapy (including allopurinol or probenecid) or placebo. Participants treated with a urate-lowering therapy experienced a highly significant reduction in BP (SBP $10.2 \mathrm{~mm} \mathrm{Hg}$ and DBP $9.0 \mathrm{~mm} \mathrm{Hg}$, respectively). Systemic vascular resistance was also reduced in the urate-lowering therapy group. ${ }^{25}$ These findings strongly supported the synergistic pathogenic role of UA and obesity in hypertension. Genes and diet are important factors affecting the levels of UA; therefore, we emphasise the importance of lifestyle modification interventions for people with prehypertension.

It was interesting that there was significant 'heterogeneity' of combined cardiovascular risk factors within the prehypertensive subgroups in our study. Compared with optimal BP, BMI and FPG were increased in the low- 
Table 3 Cardiovascular risk factors in different subranges of prehypertension

\begin{tabular}{|c|c|c|c|}
\hline & $\begin{array}{l}\text { Low-range } \\
\text { prehypertension } \\
(\mathrm{n}=925)\end{array}$ & $\begin{array}{l}\text { High-range } \\
\text { prehypertension } \\
(\mathbf{n}=894)\end{array}$ & p Value \\
\hline Age (years) & $50.6 \pm 11.2$ & $53.7 \pm 12.4^{*}$ & 0.08 \\
\hline Male (n (\%)) & $584(63.1)^{\star}$ & $579(64.8)^{*}$ & 0.47 \\
\hline Smoking (n (\%)) & $149(16.1)$ & $138(15.4)$ & 0.69 \\
\hline Alcoholic (n (\%)) & $20(1.9)$ & $22(2.7)$ & 0.29 \\
\hline Family history of hypertension (n (\%)) & $153(16.5)$ & $139(15.5)$ & 0.56 \\
\hline Overweight (n (\%)) & $183(19.8)^{*}$ & $212(23.7)^{*}$ & 0.04 \\
\hline Obesity (n (\%)) & $75(8.1)$ & $81(9.1) \dagger$ & 0.47 \\
\hline $\mathrm{BMI}\left(\mathrm{kg} / \mathrm{m}^{2}\right)$ & $23.7 \pm 3.3+$ & $26.1 \pm 3.9^{*}$ & $<0.001$ \\
\hline Dyslipidaemia (n (\%)) & $227(24.5)$ & $261(29.2)^{*}$ & 0.025 \\
\hline $\mathrm{TC}(\mathrm{mmol} / \mathrm{L})$ & $5.25 \pm 1.38$ & $5.53 \pm 1.70^{*}$ & 0.04 \\
\hline LDL-C (mmol/L) & $3.33 \pm 1.08$ & $3.58 \pm 1.19 \dagger$ & 0.10 \\
\hline $\mathrm{HDL}-\mathrm{C}(\mathrm{mmol} / \mathrm{L})$ & $1.14 \pm 0.35$ & $1.03 \pm 0.37$ & 0.69 \\
\hline TG (mmol/L) & $1.81 \pm 0.56$ & $2.34 \pm 0.67 \dagger$ & 0.12 \\
\hline $\mathrm{DM}(\mathrm{n}(\%))$ & $78(8.4)$ & $90(10.1) \dagger$ & 0.23 \\
\hline IFG (n (\%)) & $171(18.5)^{\star}$ & $213(23.8)^{\star}$ & 0.005 \\
\hline $\mathrm{FPG}(\mathrm{mmol} / \mathrm{L})$ & $5.52 \pm 1.91 \dagger$ & $6.07 \pm 2.05^{\star}$ & 0.002 \\
\hline $\operatorname{Scr}(\mathrm{mmol} / \mathrm{L})$ & $76.1 \pm 29.4$ & $73.8 \pm 27.0$ & 0.18 \\
\hline BUN (mmol/L) & $5.98 \pm 2.02$ & $6.04 \pm 1.79$ & 0.37 \\
\hline eGFR $\left(\mathrm{mL} / \mathrm{min} / 1.73 \mathrm{~m}^{2}\right)$ & $128.7 \pm 30.6$ & $121.6 \pm 28.4$ & 0.29 \\
\hline Hyperuricaemia (n (\%)) & $140(15.1)$ & $162(18.1)^{\star}$ & 0.09 \\
\hline $\mathrm{UA}(\mathrm{mmol} / \mathrm{L})$ & $392.5 \pm 40.2$ & $411.8 \pm 37.9^{\star}$ & 0.08 \\
\hline \multicolumn{4}{|c|}{$\begin{array}{l}\text { *Versus optimal blood pressure } p<0.01 \text {. } \\
\text { †Versus optimal blood pressure } p<0.05 \text {. } \\
\text { BMI, body mass index; BUN, blood urea nitrogen; DM, diabetes mellitus; eGFR, estimated glomerular filtration rate; FPG, fasting plasma } \\
\text { glucose; HDL-C, high-density lipoprotein cholesterol; IFG, impaired fasting glucose; LDL-C, low-density lipoprotein cholesterol; Scr, serum } \\
\text { creatinine: TC total cholesterol: TG trialycerides: UA serum uric acid. }\end{array}$} \\
\hline
\end{tabular}

range prehypertension group and were increased even further in the high-range prehypertension group. Furthermore, the proportions of overweight, dyslipidaemia and IFG were higher in those in the high-range prehypertension group than in those in the low-range prehypertension group. These findings confirmed the importance of the definition of prehypertension, as well as the inhomogeneity of the prehypertension subcategories. Our prior meta-analysis also found that prehypertension was associated with increased risks of $\mathrm{CVD}^{26}$ and end-stage renal disease. ${ }^{27}$ However, owing to limit prospective, randomised trials examining the effects of antihypertensive therapy on reducing target organ damage specifically in prehypertension, professional societies do not currently recommend pharmacotherapy for prehypertension, even in individuals with high-range prehypertension. $^{28} 29$ This is a great gap to be covered between epidemiological studies and randomised controlled studies in prehypertension. Prehypertensive individuals are at high risk to progress to sustained hypertension, as well as CVD and renal damage. So periodic screening is important. For therapeutic

Table 4 Multivariate logistic regression analysis for risk factors of prehypertension

\begin{tabular}{|c|c|c|c|}
\hline Risk factors & OR & $95 \% \mathrm{Cl}$ & p Value \\
\hline Age (per 10 years) & 1.21 & 1.02 to 1.44 & 0.03 \\
\hline Sex (men vs women) & 2.19 & 1.39 to 3.45 & $<0.001$ \\
\hline Smoking (yes vs no) & 0.97 & 0.70 to 1.34 & 0.85 \\
\hline Alcoholic (yes vs no) & 1.12 & 0.89 to 1.41 & 0.33 \\
\hline Family history of hypertension (yes vs no) & 1.29 & 0.88 to 1.89 & 0.19 \\
\hline Overweight/obesity (yes vs no) & 2.84 & 1.55 to 5.20 & $<0.001$ \\
\hline Dyslipidaemia (yes vs no) & 1.58 & 0.92 to 2.71 & 0.09 \\
\hline Impaired glucose regulation (yes vs no) & 1.64 & 0.96 to 2.81 & 0.07 \\
\hline Hyperuricaemia (yes vs no) & 1.70 & 1.14 to 2.54 & 0.009 \\
\hline
\end{tabular}


implications, we consider that healthcare professionals should recommend lifestyle changes to participants with prehypertension. Furthermore, high-risk subpopulations with prehypertension, especially those with high-range prehypertension, should be selected for future controlled trials to evaluate the effects of pharmacological treatment on this population.

Several limitations of this study must be considered. First, our data were based on community-based health check-up information, and not from a multistage stratified clustering sample. This may cause a bias in the prevalence of prehypertension. Second, some important confounding factors possibly associated with increased $\mathrm{BP}$, such as diet, physical activity and socioeconomic factors, were not evaluated in the present study. Third, follow-up data of individuals with prehypertension are lacking and further studies are needed on the matter.

\section{CONCLUSION}

This study showed that along with the economic development and lifestyle changes, prehypertension is highly prevalent in the Shunde District, southern China. Many other cardiovascular risk factors were present in individuals with prehypertension, especially in those with high-range prehypertension. Periodic screening and lifestyle changes should be recommended to participants with prehypertension for prevention of hypertension, as well as those with CVD.

Contributors YHua, YHu and DX conceptualised the study and designed the protocol. YHua, WQ and $\mathrm{XC}$ analysed the data and drafted the manuscript. $\mathrm{CL}$, $\mathrm{DZ}$ and $\mathrm{JH}$ collected the data. YW, YHu and DX revised the manuscript. YHu and DX participated in administrative and technical support.

Funding This research received no specific grant from any funding agency in the public, commercial or not-for-profit sectors.

\section{Competing interests None.}

Patient consent Obtained.

Ethics approval Health Authority Research Ethics Board of the First People's Hospital of Shunde, Foshan, People's Republic of China

Provenance and peer review Not commissioned; externally peer reviewed.

Data sharing statement No additional data are available.

Open Access This is an Open Access article distributed in accordance with the Creative Commons Attribution Non Commercial (CC BY-NC 4.0) license, which permits others to distribute, remix, adapt, build upon this work noncommercially, and license their derivative works on different terms, provided the original work is properly cited and the use is non-commercial. See: http:// creativecommons.org/licenses/by-nc/4.0/

\section{REFERENCES}

1. Chobanian AV, Bakris GL, Black HR, et al. The seventh report of the Joint National Committee on prevention, detection, evaluation, and treatment of high blood pressure: the JNC 7 report. JAMA 2003;289:2560-72.

2. Sun Z, Zheng L, Xu C, et al. Prevalence of prehypertension, hypertension and, associated risk factors in Mongolian and Han Chinese populations in Northeast China. Int J Cardiol 2008;128:250-4.
3. Yu D, Huang J, Hu D, et al. Prevalence and risk factors of prehypertension among Chinese adults. J Cardiovasc Pharmacol 2008;52:363-8.

4. Sun Z, Zheng L, Wei Y, et al. Prevalence and risk factors of the rural adult people prehypertension status in Liaoning Province of China. Circ J 2007;71:550-3.

5. Liu LS. [2010 Chinese guidelines for the management of hypertension]. Zhonghua Xin Xue Guan Bing Za Zhi 2011;39:579-615.

6. Elliott WJ, Black HR. Prehypertension. Nat Clin Pract Cardiovasc Med 2007;4:538-48.

7. Egan BM, Lackland DT, Jones DW. Prehypertension: an opportunity for a new public health paradigm. Cardiol Clin 2010;28:561-9.

8. Pimenta E, Oparil S. Prehypertension: epidemiology, consequences and treatment. Nat Rev Nephrol 2010;6:21-30.

9. Gupta P, Nagaraju SP, Gupta A, et al. Prehypertension-time to act. Saudi J Kidney Dis Transpl 2012;23:223-3.

10. Expert Committee on the Diagnosis and Classification of Diabetes Mellitus. Report of the expert committee on the diagnosis and classification of diabetes mellitus. Diabetes Care 2003;26(suppl 1): S5-20.

11. Joint Committee for Developing Chinese guidelines on Prevention and Treatment of Dyslipidemia in Adults. [Chinese guidelines on prevention and treatment of dyslipidemia in adults]. Zhonghua Xin Xue Guan Bing Za Zhi 2007;35:390-419.

12. Zhou B. [Predictive values of body mass index and waist circumference to risk factors of related diseases in Chinese adult population]. Zhonghua Liu Xing Bing Xue Za Zhi 2002;23:5-10.

13. Ma YC, Zuo L, Chen JH, et al. Modified glomerular filtration rate estimating equation for Chinese patients with chronic kidney disease. J Am Soc Nephrol 2006;17:2937-44.

14. Pallant J. SPSS survival manual: a step by step guide to data analysis using SPSS for windows (version 10). Open University Press, 2001.

15. Li Z, Guo X, Zheng L, et al. Prehypertension in rural northeastern china: results from the northeast china rural cardiovascular health study. J Clin Hypertens (Greenwich) 2014;16:664-70.

16. Dong GH, Wang D, Liu MM, et al. Sex difference of the prevalence and risk factors associated with prehypertension among urban Chinese adults from 33 communities of China: the CHPSNE study. $J$ Hypertens 2012;30:485-91.

17. Cheng TO. Systolic and diastolic blood pressures and urinary sodium excretion in mainland China. QJM 2000;93:557-8.

18. Tobias DK, Pan A, Jackson CL, et al. Body-mass index and mortality among adults with incident type 2 diabetes. N Engl J Med 2014;370:233-44

19. Gillman MW, Ludwig DS. How early should obesity prevention start? N Engl J Med 2013:369:2173-5.

20. Tyson CC, Appel LJ, Vollmer WM, et al. Impact of 5-year weight change on blood pressure: results from the Weight Loss Maintenance trial. J Clin Hypertens 2013;15:458-64.

21. Liang J, Xue $\mathrm{Y}$, Zou $\mathrm{C}$, et al. Serum uric acid and prehypertension among Chinese adults. J Hypertens 2009;27:1761-5.

22. Kawamoto R, Tabara Y, Kohara K, et al. Interaction between serum uric acid and triglycerides in relation to prehypertension in communitydwelling Japanese adults. Clin Exp Hypertens 2014;36:64-9.

23. Soltani Z, Rasheed K, Kapusta DR, et al. Potential role of uric acid in metabolic syndrome, hypertension, kidney injury, and cardiovascular diseases: is it time for reappraisal? Curr Hypertens Rep 2013;15:175-81.

24. Reynolds TM. Serum uric acid and new-onset hypertension: a possible therapeutic avenue? J Hum Hypertens 2014;28:519-20.

25. Soletsky B, Feig DI. Uric acid reduction rectifies prehypertension in obese adolescents. Hypertension 2012;60:1148-56.

26. Huang $\mathrm{Y}$, Wang S, Cai X, et al. Prehypertension and incidence of cardiovascular disease: a meta analysis. BMC Med 2013;11:177.

27. Huang $\mathrm{Y}$, Cai $\mathrm{X}$, Zhang J, et al. Prehypertension and incidence of ESRD: a systematic review and meta analysis. Am J Kidney Dis 2014:63:76-83.

28. James PA, Oparil S, Carter BL, et al. 2014 Evidence-based guideline for the management of high blood pressure in adults: report from the panel members appointed to the Eighth Joint National Committee (JNC 8)[J]. JAMA 2014;311:507-20.

29. Mancia G, Fagard R, Narkiewicz K, et al. 2013 ESH/ESC guidelines for the management of arterial hypertension: the Task Force for the management of arterial hypertension of the European Society of Hypertension $(\mathrm{ESH})$ and of the European Society of Cardiology (ESC). Eur Heart J 2013;34:2159-219. 